University of Nebraska - Lincoln

DigitalCommons@University of Nebraska - Lincoln

$9-1993$

\title{
Numerical study of a high-order quasiconserved quantity in the Henon-Heiles problem
}

\author{
Paul Finkler \\ University of Nebraska-Lincoln, pfinkler1@unl.edu \\ C. Edward Jones \\ University of Nebraska-Lincoln \\ Glenn A. Sowell \\ University of Nebraska at Omaha
}

Follow this and additional works at: https://digitalcommons.unl.edu/physicsfinkler

Part of the Physics Commons

Finkler, Paul; Jones, C. Edward; and Sowell, Glenn A., "Numerical study of a high-order quasiconserved quantity in the Henon-Heiles problem" (1993). Paul Finkler Papers. 8.

https://digitalcommons.unl.edu/physicsfinkler/8

This Article is brought to you for free and open access by the Research Papers in Physics and Astronomy at DigitalCommons@University of Nebraska - Lincoln. It has been accepted for inclusion in Paul Finkler Papers by an authorized administrator of DigitalCommons@University of Nebraska - Lincoln. 


\title{
BRIEF REPORTS
}

Brief Reports are accounts of completed research which do not warrant regular articles or the priority handling given to Rapid Communications; however, the same standards of scientific quality apply. (Addenda are included in Brief Reports.) A Brief Report may be no longer than 4 printed pages and must be accompanied by an abstract. The same publication schedule as for regular articles is followed, and page proofs are sent to authors.

\section{Numerical study of a high-order quasiconserved quantity in the Hénon-Heiles problem}

\author{
Paul Finkler and C. Edward Jones \\ Department of Physics and Astronomy, University of Nebraska-Lincoln, Lincoln, Nebraska 68588-0111 \\ Glenn A. Sowell \\ Department of Physics, University of Nebraska at Omaha, Omaha, Nebraska 68182-0266
}

(Received 5 April 1993)

\begin{abstract}
Recent efforts to derive and study a quasiconserved quantity $K$ in the Hénon-Heiles problem in terms of a single set of variables are discussed. Numerical results are given, showing how the value of such a quantity varies with time and order in a power-series expansion for $K$ in terms of monomials of the coordinates and velocities. The lowest order in the power series for $K$ corresponds to $n=4$ and the highest order to $n=27$, so that 24 orders are included in the series. The results are compared with an earlier study by the authors [Phys. Rev. A 42, 1931 (1990)] that included an expansion for $K$ for orders $n=4$ to $n=15$. In general, even in regions where the earlier study suggested that the series for $K$ might be converging, our more recent results [Phys. Rev. A 44, 925 (1991)], involving twice as many orders, suggest that the series diverges.

PACS number(s): 05.45. $+\mathrm{b}, 03.20 .+\mathrm{i}, 46.10 .+\mathrm{z}, 95.10 . \mathrm{Ce}$
\end{abstract}

\section{INTRODUCTION}

This paper updates an ongoing study $[1,2]$ of a quasiconserved quantity $K$ (in addition to the energy) in the well-known two-dimensional Hénon-Heiles problem [3]. The quality $K$ is expressed as a formal power series in the $x$ and $y$ coordinates and velocities. The original work by Gustavson [4] on this subject provided a method for determining such a $K$ using canonical transformations and normal forms. Truncating the series after five orders $(n=4-8)$, Gustavson showed numerically that surfaces of constant $K$ approximated the one-dimensional curves in the Poincaré sections characteristic of nonchaotic motion. Subsequently, Shirts and Reinhardt [5] calculated ten orders in the series for $K$ using the Gustavson technique [4] and then converted the expression into a Padé approximant, which appeared to have poles corresponding to points in phase space where trajectories were chaotic.

The present authors' interest in studying $K$ centers around its use as a possible tool in understanding the approach to chaos. As mentioned above, surfaces of constant $K$ (obtained by truncating the formal power series) produce Poincaré sections which approximate those calculated directly for nonchaotic trajectories. We had devised a method $[1,2]$ for determining the formal series for $K$ without the cumbersome and repeated use of canonical transformations (we refer to Ref. [1] as FJS1 and Ref. [2] as FJS2). The goal was to develop a method where one could generate many orders in the expansion of $K$ in hopes of gaining insight from numerical studies about the convergence properties of the power-series expansion for $K$. Does the series offer any tell-tale signs when going from regions where the motion is nonchaotic to regions where it is chaotic? Could it be convergent at any points in phase space?

In FJS1, a method was developed for determining $K$ using a single set of variables without employing canonical transformations and a total of 12 orders $(n=4-15)$ were calculated for $K$. It was evaluated numerically for a number of trajectories and plotted as a function of time, truncating the series at various orders to gain some feeling for the convergence and constancy in time of the series. These results are summarized in Figs. 1-5 of FJS1. For certain trajectories at lower energies, $K$ appears to be converging and is reasonably constant over the time interval selected of $100 \mathrm{~s}$. At higher energies in regions where the trajectories are known to be chaotic, the series appears neither to converge nor to be constant.

In FJS2, a more powerful and streamlined technique was introduced for determining a power series for $K$, again using a single set of variables. Using this method, we have determined $K$ to 24 orders or twice as many as considered in FJS1. We discuss here how these extra terms modify the results given in FJS 1 and further clarify the apparent convergence properties of the series for $K$.

In Sec. II, we briefly review the technique of FJS2 for generating the series for $K$. In Sec. III, we give graphs of 
$K$ versus time $t$ for the same trajectories used in FJS1 which show the effect of including twice as many orders in the $K$ expansion.

\section{SERIES FOR $K$}

The Hënon-Heiles problem involves motion in two dimensions with the following kinetic energy $T$ and potential energy $U$ :

$$
\begin{aligned}
& T=\frac{1}{2}\left(\dot{x}^{2}+\dot{y}^{2}\right), \\
& U=\frac{1}{2}\left(x^{2}+y^{2}\right)+x^{2} y-\frac{1}{3} y^{3} .
\end{aligned}
$$

In FJS2, the following four complex variables are introduced:

$$
\begin{aligned}
& \beta=(x+i y)+i(\dot{x}+i \dot{y}), \\
& \beta^{*}=(x-i y)+i(\dot{x}-i \dot{y}), \\
& \bar{\beta}=(x+i y)-i(\dot{x}+i \dot{y}), \\
& \bar{\beta}^{*}=(x-i y)-i(\dot{x}-i \dot{y}),
\end{aligned}
$$

One assumes a constant of the motion $K$ (independent of the energy), which has a power-series expansion in terms of the variables just defined, as follows:
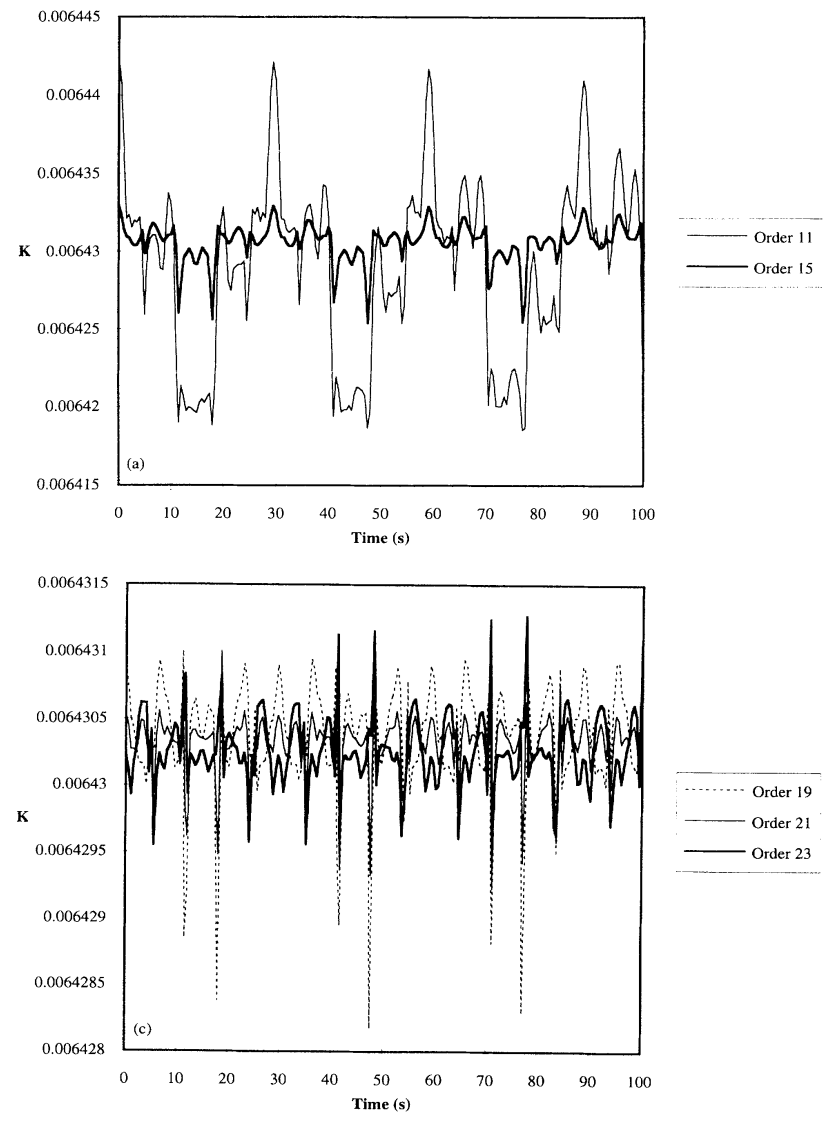

$$
K\left(\beta, \beta^{*}, \bar{\beta}, \bar{\beta}^{*}\right)=\sum_{n=n_{0}}^{\infty} K_{n},
$$

where $K_{n}$ is an $n$ th-order term in the $\beta$ variables and $n_{0}=4$. The detailed procedure for determining $K_{n}$ is given in FJS2 and consists of solving iteratively the differential equations

$$
\begin{aligned}
& D_{0} K_{n_{0}}=0, \\
& D_{0} K_{n+1}=D_{1} K_{n},
\end{aligned}
$$

where $D_{0}$ and $D_{1}$ are differential operators derived and discussed in FJS2. If (2.8) and (2.9) are satisfied, $K$ in (2.7) is formally a conserved quantity.

\section{NUMERICAL RESULTS FOR $\boldsymbol{K}$}

We have determined the terms in the series (2.7) for $K$ for $n=4-27$, a total of 24 orders, using a computer algebra system [6]. We then evaluated $K$ along the same representative trajectories as in FJS1, truncating the series at various orders so as to compare with the numerical results of the earlier paper. Although the procedure for developing the $K$ series in FJS1 is somewhat different than in FJS2 (including having different $n=4$ lowest-
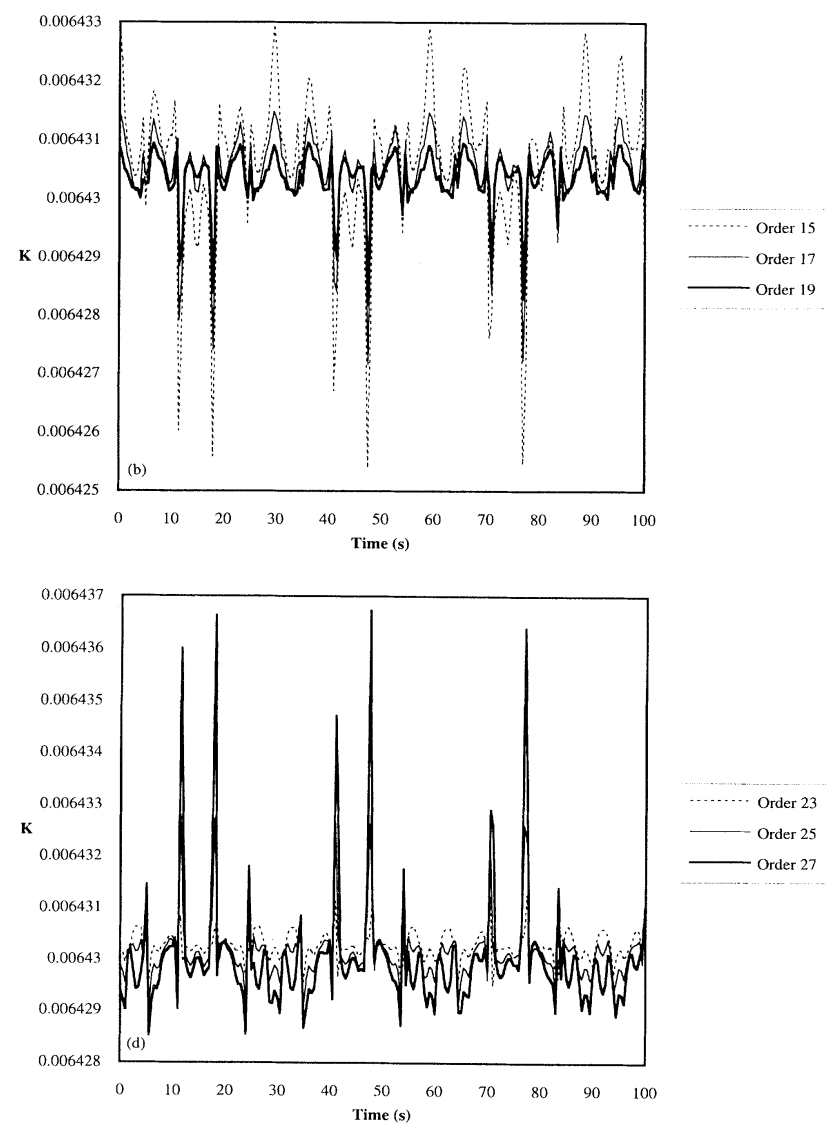

FIG. 1. Graphs of the series $K$. The energy is $E=0.05$ with the initial conditions $x=\dot{y}=0, y=-0.15$, and $\dot{x}$ negative. (a) Graph of the series $K$ through 11 th and 15th orders. (b) Graph of the series $K$ through 15 th, 17 th, and 19th orders. (c) Graph of the series $K$ through 19th, 21st, and 23rd orders. (d) Graph of the series $K$ through 23rd, 25th, and 27th orders. 
order terms in the two cases), this, as we expected, does not change the apparent structure of the convergence of the series through a given order in the two cases as we shall see. For the lowest-order term in (2.7) in the present calculation, we have taken the simple and symmetric term

$$
K_{4}=\beta \beta^{*} \bar{\beta} \bar{\beta}^{*} \text {. }
$$

In Fig. 1, we show the results for $100 \mathrm{~s}$ for the trajectory with energy $E=0.05$ and initial conditions $x=\dot{y}=0$, $y=-0.15$, and $\dot{x}$ (whose magnitude is fixed by the other quantities) taken to be negative. This trajectory corresponds to one considered in FJS1 in Fig. 1 of that paper. Here Fig. 1(a) gives $K$ vs $t$ when the series is truncated after order $n=11$ and 15 (i.e., the $K$ series summed from $n=4-11$ and $n=4-15)$. This graph can be compared directly to Figs. 1(b) and 1(c) of FJS1. Although the value of $K$ is different (due to a different construction algorithm and starting point for the series), we see that the convergence properties are very similar. The Poincare section given in Fig. 1(a) of FJS1 suggests that this is a nonchaotic trajectory. And, indeed, the graphs are consistent with a converging series for $K$.

In Figs. 1(b), 1(c), and 1(d), the curve of $K$ vs $t$ is shown with the $K$ series truncated after orders $n=15,17$, and 19, after orders $n=19,21$, and 23 and after orders
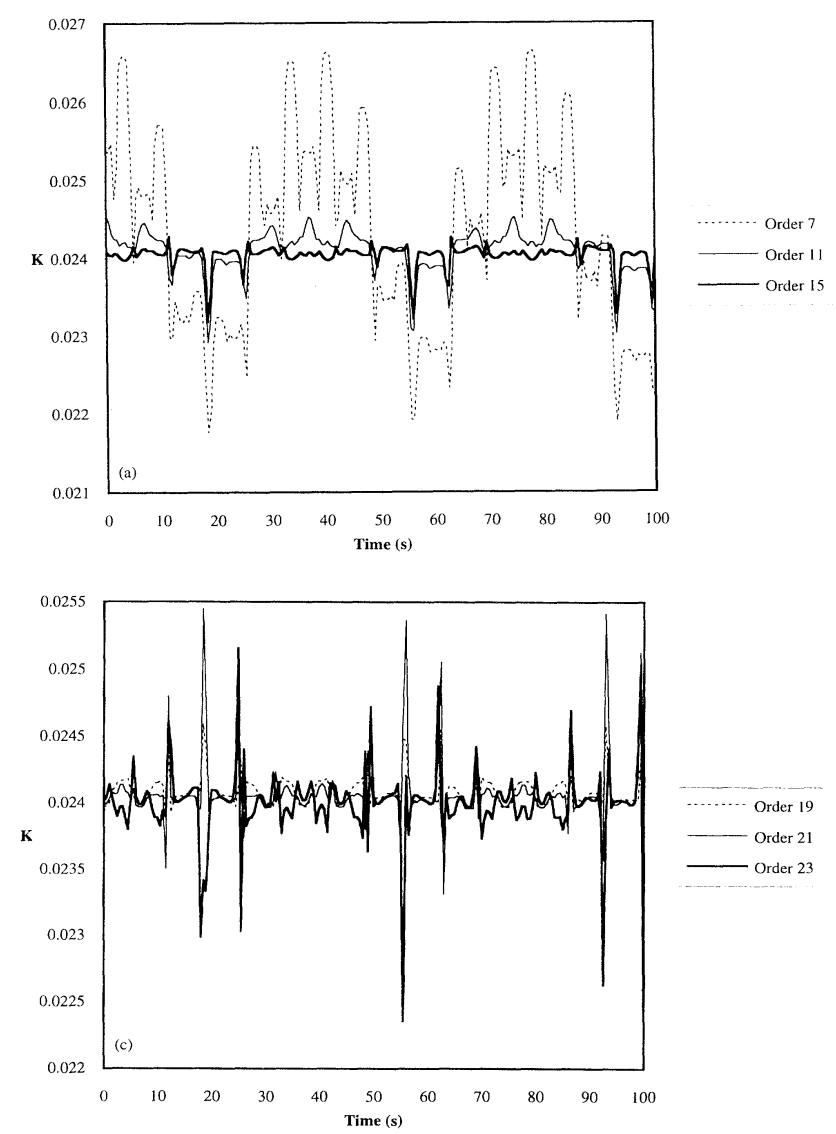

$n=23,25,27$, respectively. Figure $1(\mathrm{~b})$ is still consistent with the idea of a convergent $K$ series but Figs. 1(c) and 1(d) show a developing tendency for the series to diverge.

In Fig. 2, we examine the corresponding trajectory to that in Fig. 2 of FJS1. Here the energy is $E=0.08$ and the other initial conditions are the same as before ( $x=\dot{y}=0, y=-0.15$, and $\dot{x}$ negative). Figure 2(a) shows $K$ vs $t$ for $100 \mathrm{~s}$ truncated after orders $n=7,11$, and 15 and Figures. 2(b), 2(c), and 2(d) follow the same pattern as for the previous trajectory. We see that Fig. 2(a) is very similar to Figs. 2(b) and 2(c) of FJS1, showing a possible convergent series. Figures 2(b), 2(c), and 2(d), on the other hand, show a growing divergence as more terms are added to the series. When all orders are included through $n=27$ [see Fig. 1(d)], $K$ shows large fluctuations as a function of $t$ and actually changes sign.

\section{DISCUSSION}

The results in Figs. 1 and 2 appear to indicate that as more terms are kept in the expansion for $K$, the series will probably diverge even in regions of phase space where the trajectory may not be chaotic. Of course, we know from Gustavson's work that surfaces of constant $K$ reproduce approximately the Poincare section curves, so the series for $K$ clearly contains some physical informa-
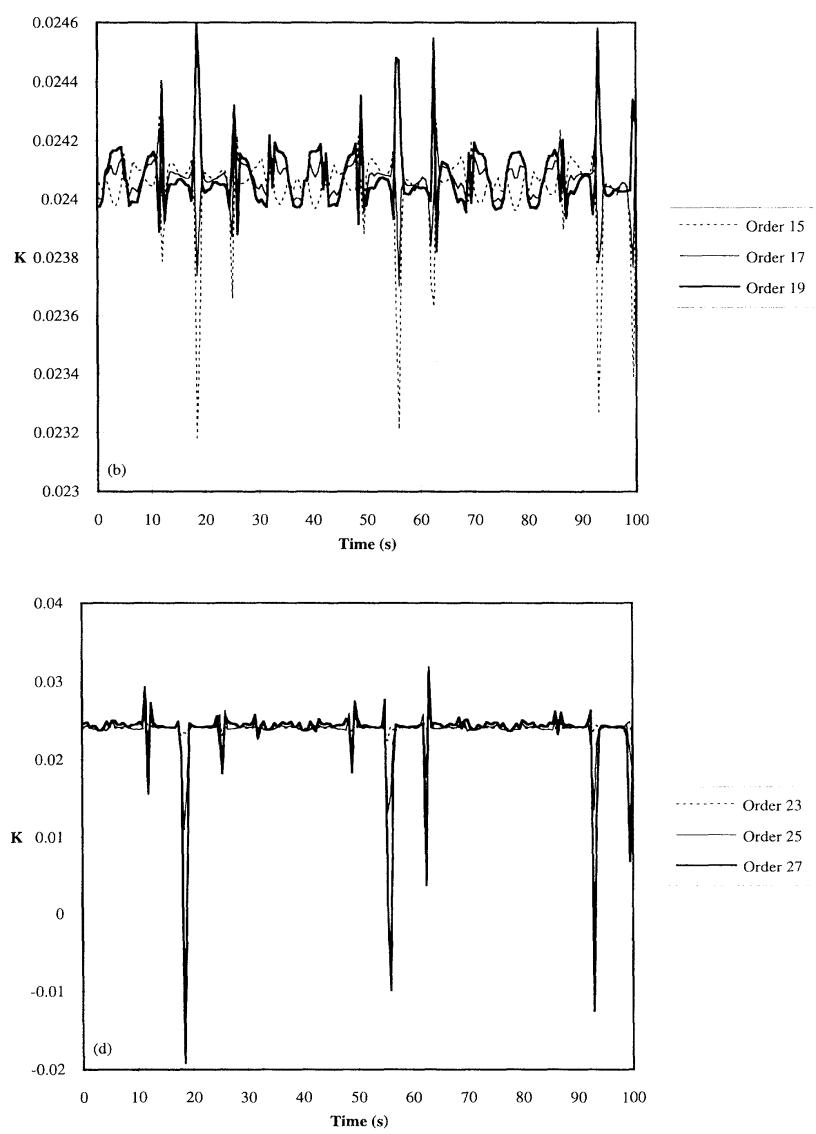

FIG. 2. Graphs of the series $K$. The energy is $E=0.08$ with the initial conditions $x=\dot{y}=0, y=-0.15$, and $\dot{x}$ negative. (a) Graph of the series $K$ through 11 th and 15th orders. (b) Graph of the series $K$ through 15 th, 17th, and 19th orders. (c) Graph of the series $K$ through 19th, 21st, and 23rd orders. (d) Graph of the series $K$ through 23rd, 25th, and 27th orders. 
tion, even though it may not, as indicated here, be convergent. It is of interest to ask whether the series for $K$ might be an asymptotic one and also whether there are any points in phase space where it converges. Some insight on these questions can be obtained by transforming the series into Padé approximants as was first done by Shirts and Reinhardt [5]. Such approximants can, in some cases, provide expressions that are more accurate than the original power series, while using the same information. Using the information from such series with our 24 terms will enable us to extend the work of Shirts and Reinhardt [5] and provide more insight into its convergence properties. This work will be reported on elsewhere.
[1] P. Finkler, C. E. Jones, and G. A. Sowell, Phys. Rev. A 42, 1931 (1990), hereafter referred to as FJS1.

[2] P. Finkler, C. E. Jones, and G. A. Sowell, Phys. Rev. A 44, 925 (1991), hereafter referred to as FJS2.

[3] M. Hénon and C. Heiles, Astron. J. 69, 73 (1964).

[4] F. G. Gustavson, Astron. J. 71, 670 (1966).
[5] R. B. Shirts and W. P. Reinhardt, J. Chem. Phys. 77, 5204 (1982).

[6] In FJS1 we used MACSYMA from Symbolics, Inc. In FJS2 and this paper we have used MAPLE from Waterloo Maple Software, Inc. 